\section{Observing our psychiatric practice}

Medicine is defined by both cultural perception and scientific discovery. As we depart from the era of paternalism, we face modern practice: we value reflective learning, measure quality of life and advocate patient-centred care. I understand this through my observational learning as a medical student. Now in my final year, my most alarming memories relate to the mounting challenge of mental healthcare.

Psychiatry is a culturally growing, financially shrinking specialty. The medical profession acknowledges the huge burden of mental illness, but a subtle stigma remains in our community. A primary shortcoming in our care lies in our attitude; we hold preconceptions about mental health patients, undermine the medical expertise of our psychiatrists and neglect our own mental well-being. From the alcoholic with suicidal intent in the emergency room, to the widow with anxiety in general practice, these 'time-wasters' will be greeted, eventually, with angry mutterings and extended waiting times. There is no quick fix for these patients, and after our failed prevention strategies, the best treatment we appear to offer is an off-site psychiatric hospital.

The negative perception strikes again during career choice discussions, where any enthusiasm for this specialty is battered down with a distinct mixture of mockery and disappointment: 'But why do you want to give up real medicine?'. The separation between mental and physical health occurs early in our medical training - immediately after the second foundation year. I question the potential prematurity of this divide - particularly from its counterpart of neurology - and speculate on the role that this plays in its underfilled training programme and undervalued perceptions.

The stereotypes of each specialty can be comical. The danger in psychiatry is that patient safety is being jeopardised. Mental healthcare is inseparable from medical practice; it coexists in every physical health consultation, every patient journey, as well as dictating our own practice. This synergism is not reflected in our curricula - a pertinent example of this includes the omission of a patient's mental state in a routine history. This ignorance escalates into our unconscious clinical decision-making, where psychiatric diagnoses become an afterthought or diagnosis of exclusion. I have witnessed countless cases of this: a 6-year-old boy presenting with generalised abdominal pain urgently operated on for surgical exploration, before discovering his extensive anxiety from school bullying and a violent home environment. The appropriate referral for family therapy helped alleviate his regular pain as well as the underlying causative factors. Our lack of integration in care also appears at the tertiary level: in one asthma clinic, an anxious young woman on her third referral for breathing difficulties was offered an invasive procedure to reassure her 'likely anxiety'. The distinct overlap between mental and physical health - notably in specialties such as gastroenterology and gynaecology - is fascinating, but our denial through the constrained use of psychological services is frustrating. We are diminishing our patients' quality of life, encouraging deterioration in physical health and fuelling public stigma against mental illness.

Greater change in our attitude and practice is requisite to achieving the principles of our modern medicine. Despite the financial and logistical challenges we currently face in our healthcare system, a vast number of holistic, open-minded clinicians remain exempt from these anecdotes. They prove to me that these changes are possible, and motivate the next generation of doctors to speak out against the medical hierarchy.

Adisha Kapila, Medical Student, GKT School of Medical Education, King's College London, UK. Email: adisha.kapila@kcl.ac.uk

doi: $10.1192 /$ bjp.211.4.252

\title{
Correction
}

Staff experiences and perceptions of working with in-patients who are suicidal: qualitative analysis. BJPsych, 211, 103-108. The Funding paragraph (p. 107) should read: This paper presents independent research funded by the National Institute for Health Research (NIHR) under its Research for Patient Benefit (RfPB) Programme (Grant Reference Number PB-PG-1111-26026). The views expressed are those of the authors and not necessarily those of the NHS, the NIHR or the Department of Health.

doi: 10.1192/bjp.211.4.252a 